\title{
TAIPAN: Optical Spectroscopy with Starbugs
}

\author{
Kyler Kuehn ${ }^{a}$, Jon Lawrence ${ }^{a}$, David M. Brown ${ }^{a}$, Scott Case ${ }^{a}$, Matthew Colless ${ }^{b}$, Robert \\ Content $^{a}$, Luke Gers ${ }^{a}$, James Gilbert ${ }^{a, c}$, Michael Goodwin ${ }^{a}$, Andrew M. Hopkins ${ }^{a}$, Michael \\ Ireland $^{b}$, Nuria P. F. Lorente ${ }^{a}$, Rolf Muller ${ }^{a}$, Vijay Nichani ${ }^{a}$, Azizi Rakman ${ }^{a}$, Samuel N. \\ Richards $^{d}$, Will Saunders ${ }^{a}$, Nick F. Staszak ${ }^{a}$, Julia Tims ${ }^{a}$, Lewis G. Waller ${ }^{a}$ \\ ${ }^{a}$ Australian Astronomical Observatory, 105 Delhi Rd, North Ryde, NSW, 2113, Australia \\ ${ }^{b}$ Australian National University, Canberra, ACT, 0200, Australia \\ ${ }^{c}$ University of Oxford, Keble Road, Oxford OX1 3RH, United Kingdom \\ ${ }^{d}$ University of Sydney, Sydney, NSW, 2006, Australia
}

\begin{abstract}
TAIPAN is a spectroscopic instrument designed for the UK Schmidt Telescope at the Australian Astronomical Observatory. In addition to undertaking the TAIPAN survey, it will serve as a prototype for the MANIFEST fibre positioner system for the future Giant Magellan Telescope. The design for TAIPAN incorporates up to 300 optical fibres situated within independently-controlled robotic positioners known as Starbugs, allowing precise parallel positioning of every fibre, thus significantly reducing instrument configuration time and increasing observing time. We describe the design of the TAIPAN instrument system, as well as the science that will be accomplished by the TAIPAN survey. We also highlight results from the on-sky tests performed in May 2014 with Starbugs on the UK Schmidt Telescope and briefly introduce the role that Starbugs will play in MANIFEST.
\end{abstract}

Keywords: Astronomical Instrumentation, High Multiplex and Survey Instruments, Fiber Positioner Technology, Spectroscopy

\section{INTRODUCTION}

Currently, astronomical multi-object spectroscopy (MOS) uses technologies such as fixed-position plug plates (e.g., SAMI ${ }^{1}$ SDSS-III ${ }^{2}$ ) or pick and place robots that position fibres serially (e.g., $2 \mathrm{dF}^{3}$ ). Even the fastest of these require tens of minutes to an hour to prepare a full complement of astrophysical target observations; despite the constraints of such systems, however, observations utilizing such technologies have been very successful. Nevertheless, the astronomical community has long sought methods for overcoming the deficiencies of these systems - particularly the potential time lost from observing, as well as the lack of flexibility in observations once targets have been selected.

Several technologies to enable massive parallelisation of fibre positioning have been developed at the Australian Astronomical Observatory (AAO) and affiliated institutions, including "tilting spines" ${ }^{4}$ and Starbugs. ${ }^{5}$ It is the latter technology, along with its initial application in an astronomical context, that we discuss in this paper. Section 2 discusses the limits of currently-existing fibre-positioning technologies and the design and capabilities of Starbugs, while Section 3 describes the TAIPAN system - a multi-object spectroscopy instrument with Starbugs playing a central role. Section 4 describes the science programs to be executed with the TAIPAN instrument, including the TAIPAN galaxy survey and the FunnelWeb stellar survey. Section 5 describes the datasets required for these surveys, and the operation method (in particular the Starbug target allocation, routing, and motion control) that will allow these datasets to be acquired. Section 6 describes the test program (dubbed Proto-TAIPAN) recently completed with several prototype Starbugs installed at the focal plane of the UK Schmidt Telescope (UKST). We conclude in Section 7 with a look ahead to the MANIFEST many-fibre system that will be installed on the Giant Magellan Telescope (GMT) within the next decade. ${ }^{6,7}$

Further author information: (Send correspondence to K.K.)

K.K.: E-mail: kyler.kuehn@aao.gov.au, Telephone: +61 293724889

Ground-based and Airborne Instrumentation for Astronomy V, edited by

Suzanne K. Ramsay, lan S. McLean, Hideki Takami, Proc. of SPIE Vol. 9147,

$914710 \cdot$ (C) 2014 SPIE · CCC code: $0277-786$ X/14/\$18 · doi: 10.1117/12.2055677

Proc. of SPIE Vol. 9147 914710-1 


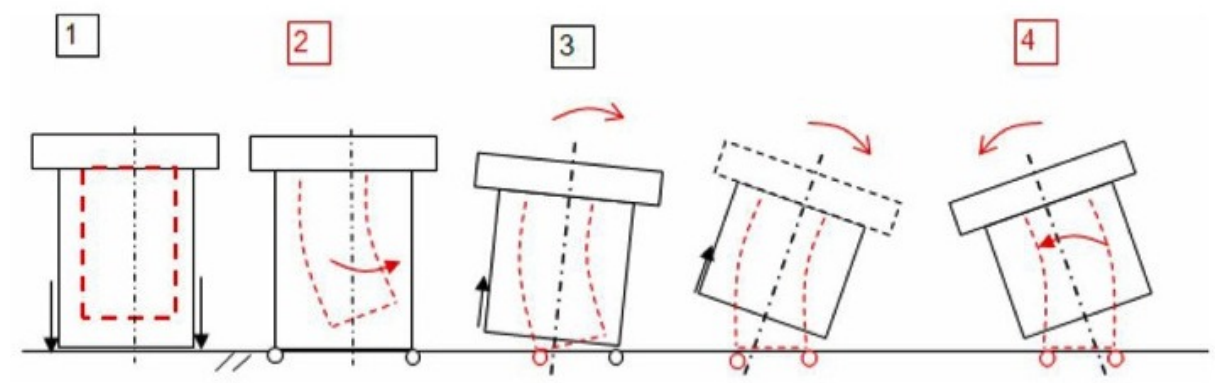

Figure 1. The coordinated motion of the concentric piezoceramic tubes provides the Starbug "walking" motion.

\section{MULTI-OBJECT SPECTROSCOPY WITH OPTICAL FIBRES}

Multi-object spectroscopy (MOS) primarily uses fixed-position plates (e.g., SAMI, ${ }^{1}$ SDSS-III ${ }^{2}$ ) or pick-and-place robots that position fibres serially (e.g., $2 \mathrm{dF}^{3}$ ), often taking tens of minutes to an hour to prepare a field for observation. These have been very successful, given the constraints of the system. But in addition to potential time lost from observing, this lengthy preparation process makes an entire observing program much less flexible - for example, Targets of Opportunity are often impossible to follow up.

What is needed is parallelisation of the fibre positioning. One enabling technology for this technique that has been developed at the AAO is Starbugs. The Starbug system consists of concentric piezo-ceramic tubes that contract and expand or flex to allow a stepping motion across a surface (Figure 1). These tubes can then be outfitted with an optical fibre payload in their common centre, and placed on a transparent surface at the focal plane of a telescope. Such a fibre can be positioned to within a few microns, allowing positioning accuracy to better than an arcsecond. ${ }^{8}$ The basic walking motion has been demonstrated previously, ${ }^{5}$ but refinements in the design and construction of the bugs were necessary for consistent, repeatable performance adequate to the task of rapid movement, micron-level positioning accuracy, and payload deployment. Details of these design and construction improvements, and a description of the concomitant performance improvements, are provided in Ref. 9.

\section{TAIPAN}

Around the enabling technology of Starbugs, we are building the TAIPAN spectroscopic instrument. The optical fibre payloads of the TAIPAN Starbugs will allow us to engage in the TAIPAN (Transforming Astronomical Imaging surveys through Polychromatic Analysis of Nebulae) survey on the UK Schmidt Telescope at Siding Spring Observatory starting in 2016. This survey will incorporate 150 Starbugs (initially, with a potential upgrade to 300) and will cover at least $20 \mathrm{~K} \mathrm{sq.} \mathrm{deg.} \mathrm{over} \mathrm{the} \mathrm{southern} \mathrm{hemisphere,} \mathrm{targeting} \mathrm{of} \mathrm{order} 500 \mathrm{~K}$ galaxies. Several key scientific results will emerge from this survey dataset, particularly related to cosmology and galaxy evolution. The FunnelWeb survey will use the TAIPAN instrument to observe the same sky area, but will target stars instead of galaxies. This survey will attain a nearly complete survey for all stars in the magnitude range $5<\mathrm{V}<12$. Section 4 provides further details of the scientific goals of these two surveys.

The TAIPAN Starbugs are held with vacuum onto a glass field plate matching the focal surface of the UKST. When high-voltage waveforms are applied in the proper form and sequence to the piezoceramic tubes, the Starbugs move at a rate of $\sim 2 \mathrm{~mm} / \mathrm{s}$. A 29 Megapixel metrology camera situated in the central hole of the UKST primary mirror views the entire focal plane, and measures the locations of each Starbug, providing feedback as necessary to the Starbug control system. When all of the Starbugs are positioned properly, light will enter the optical fibre payloads of the Starbugs and then travel $\sim 10 \mathrm{~m}$ to feed an $\mathrm{R} \sim 2300$ spectrograph, a schematic of which is shown in Figure 2. The spectrograph makes use of two $2 \mathrm{k} \times 2 \mathrm{k}$ CCDs manufactured by $\mathrm{e} 2 \mathrm{~V}$, one with a coating optimised for sensitivity in the blue $(370-577 \mathrm{~nm})$ and another with a coating optimised for sensitivity in the red $(557-870 \mathrm{~nm})$. The throughput for the entire system, from the atmosphere through the telescope down the fibres and into the spectrograph, is shown as a function of wavelength in Figure 3. 


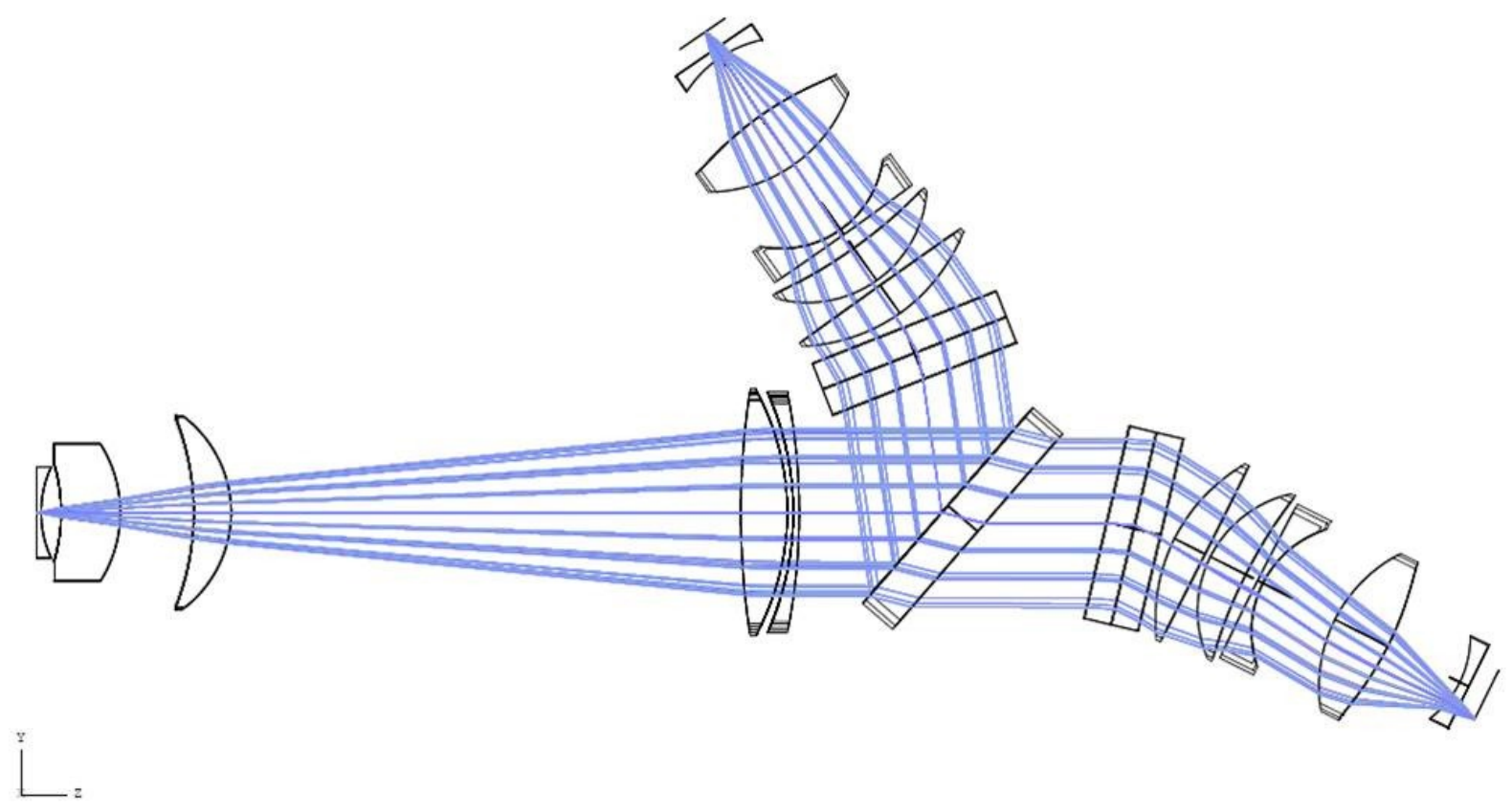

Figure 2. The TAIPAN Spectrograph. Light enters from the left and is divided at $567 \mathrm{~nm}$ by the beamsplitter (centre). The light is then focused into two different CCDs, one sensitive to blue wavelengths (top) and one sensitive to red wavelengths (right).

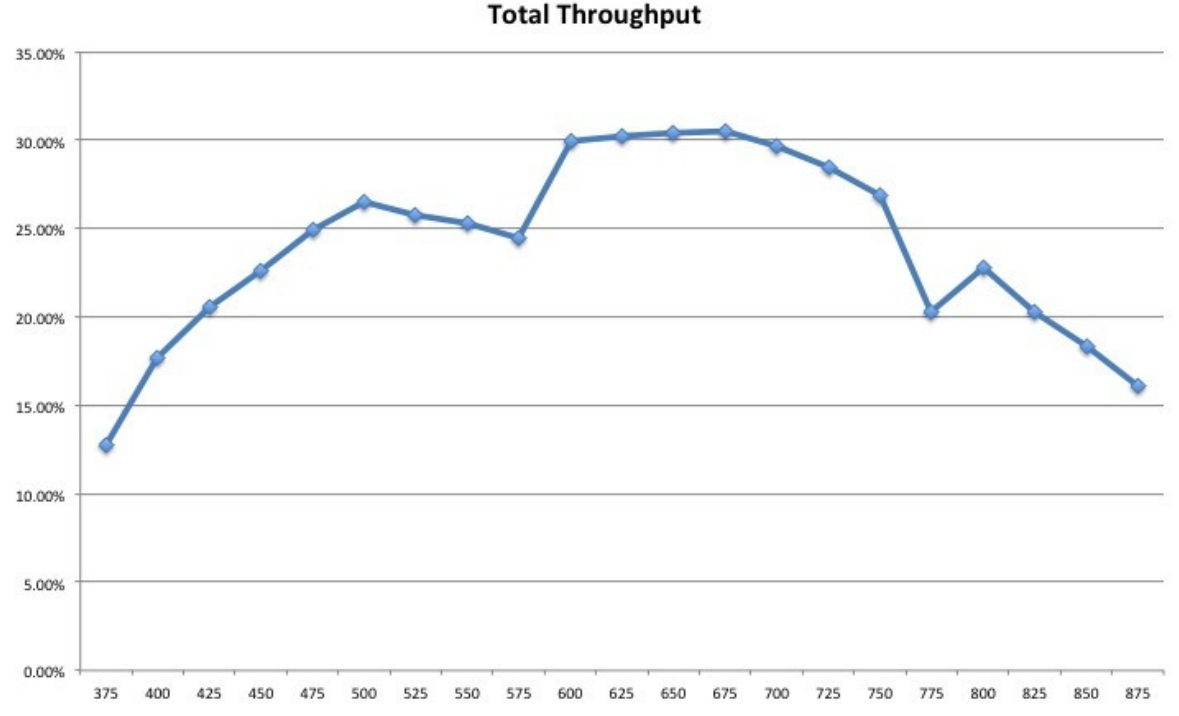

Figure 3. Estimated system throughput for TAIPAN as a function of wavelength, including the atmosphere, the telescope and instrument optics, fibre losses, and CCD quantum efficiency. The throughput for TAIPAN is more than a factor of 2 improved over $6 \mathrm{dF}$, the previous instrument that was installed on the UK Schmidt Telescope. 


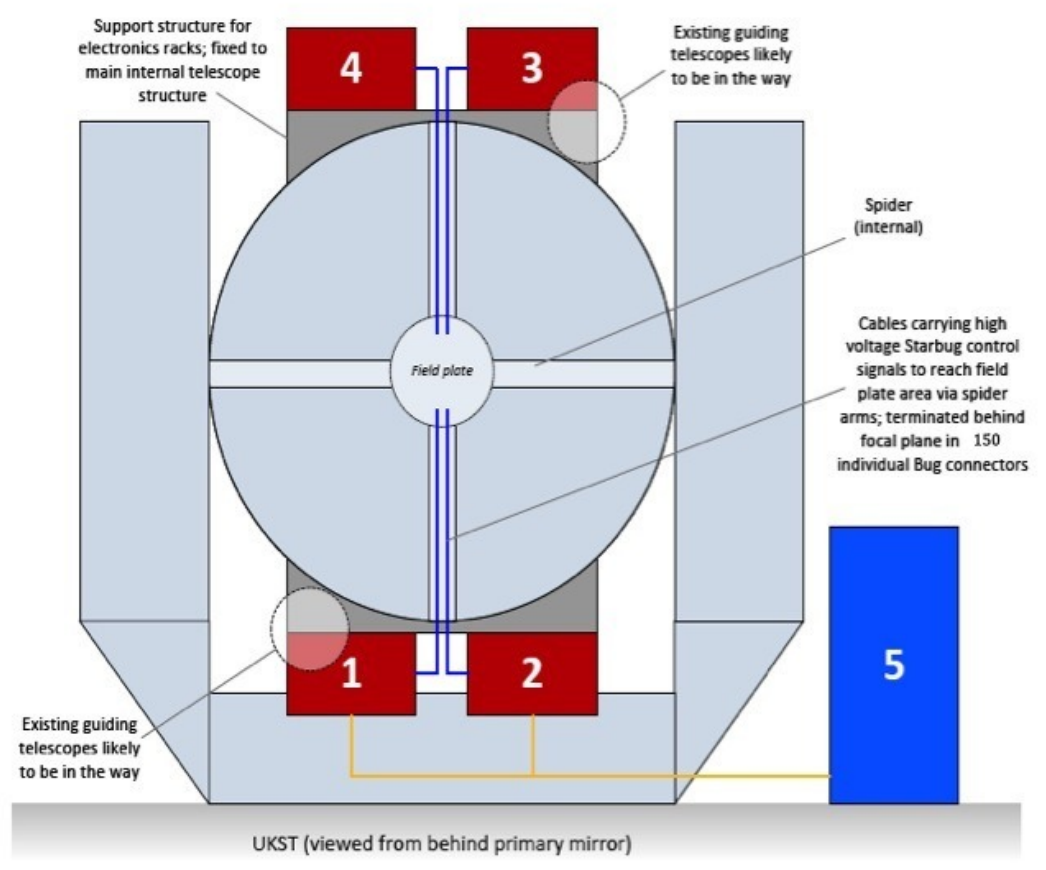

Figure 4. A schematic of the UKST structure with the TAIPAN field plate, along with the basic routing for its electrical power and data connections. 1-4 are electronics and control racks for 75 Starbugs each, 5 is the high voltage power source located on the floor of the UKST dome.

In addition to the new instrument to be installed on the UK Schmidt Telescope, the UKST itself will undergo significant infrastructure upgrades, particularly to the dome/shutter/windscreen drive motors and controls, the telescope control system (including motors, controls, and software), the telescope and instrument user interface, and the telescope guiding system. A schematic of the UKST with components of the new instrument and power distribution system is shown in Figure 4.

\section{SCIENCE GOALS AND REQUIREMENTS OF THE TAIPAN SURVEY}

As mentioned above, TAIPAN will observe $\sim 500 \mathrm{~K}$ galaxies, to a magnitude of $\sim 17.5$ with a Signal to Noise goal of 5-10 across the entire southern hemisphere. TAIPAN will explore the formation history and evolution of these galaxies throughout cosmic time (to at least $\mathrm{z} \sim 1$, and will precisely map their radial velocities of as a function of position and distance. The primary cosmology goal of the TAIPAN survey is to determine the value of $\mathrm{H}_{0}$ to $\sim 1 \%$, though the dataset will be used for far more than just this purpose. For example, it will enable studies of the connection between gas and stars, the impact of environment and mergers on galaxy evolution, large scale structure (including stellar and halo mass function), star formation activity correlated with AGN activity, and (in conjunction with radio surveys performed with telescopes such as ASKAP ${ }^{10}$ ) the intergalactic magnetic field.

FunnelWeb, the stellar survey using the TAIPAN instrument, will provide a nearly complete $(>99 \%)$ census of all stars in the magnitude range $5<\mathrm{V}<12$ in the southern hemisphere ( $>2 \mathrm{M}$ targets with a Signal to Noise of 100 or more). These will be used to search for exoplanets directly in the FunnelWeb data, and indirectly by providing a well-calibrated input catalog to other missions such as TESS. ${ }^{11}$ Additionally, FunnelWeb will provide highly accurate measurements relevant to astroseismology, stellar variability, galactic archaeology, astrometry (including stellar proper motions), calibration standards, radial velocities, and spectral line widths (thereby allowing for the determination of stellar metallicity, surface gravity, and similar characteristics). Studies of the intervening ISM (by mapping the reddening of stellar light vs. position and distance) will also be done.

We need to not only observe a half million galaxies and 2 million stars during the survey, but these data must have sufficient signal to noise and sufficient wavelength stabilty in order for the science teams to realise 


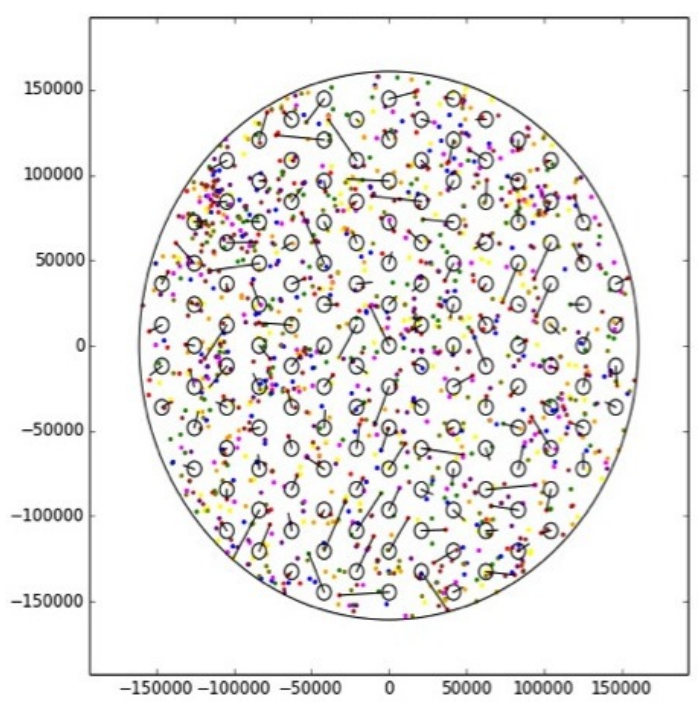

Figure 5. A single TAIPAN target field, 6 degrees in diameter. The individual targets are shown as the small filled circles, while the Starbugs are the depicted by the larger open circles. The Starbug paths to the targets selected by the Allocator are shown by solid lines.

their goals. This places stringent requirements on the optomechanical components, the spectrograph and its environment, and the Starbugs themselves. For example, the science fibre payload must be aligned within the Starbug to within 1 degree, lest the light enter the fibre off-axis and cause degradation of the signal. Similarly, the spectrograph temperature must be kept stable to within 1 degree, lest the positions on the CCDs correlated with specific wavelengths via calibration observations shift. These and a whole host of other engineering and performance requirements have been produced and are being tested against prototypes of each component, and, where applicable, in an integrated system (see Section 6, below).

\section{TAIPAN OPERATIONS}

To cover 20K sq. deg. (with multiple passes) in 5 years, we must observe of order 1000 galaxies per night in dark and gray time. To accomplish this overall plan, we will observe galaxies for 2.5 hours on a given field, split into a series of 30 min exposures that are then co-added. Because of their rapid reconfiguration time, Starbugs can be dynamically allocated to targets inbetween exposures - with this method, brighter targets will be observed only for a single 30 min exposure, and then new targets are acquired, while the Starbugs positioned to observe fainter objects remain in place for the subsequent 30 minute exposure(s). Dynamic allocation allows approximately $3 \times$ the base number of targets to be observed in a 2.5 hour period (i.e., 150 Starbugs will yield 450 targets). A sample target galaxy field (with Starbugs allocated to individual targets) is shown in Figure 5. The FunnelWeb stellar survey provides an even greater observational challenge, as we must observe of order 5000 stars in bright time every night. This will be accomplished with significantly shorter (2-3 minute) exposures, since these targets are many mangitudes brighter than the galaxy targets.

To observe these galaxies and stars, we must position an optical fibre payload from a Starbug on each of them. The Starbug motion control algorithm begins with the Allocation of each Starbug to a target, and proceeds through the Routing stage, which determines the best route from the Starbugs' current positions to their next target position (taking into account collision avoidance and minimisation of the total reconfiguration time). Finally, the Motion stage sees each Starbug actually undertake the change in position dictated by the Routing algorithm, with feedback to a Metrology system that allows real-time correction of Starbug trajectories, with the final outcome being the positioning of each starbug to within a few microns (or about $1 / 20$ th of a pixel). ${ }^{8}$ While a 5 minute reconfiguration is required time to successfully complete the survey in the allotted 5 years, an improvement to 2-3 minutes for reconfiguration would improve the survey efficiency dramatically; thus we are continually refining the steps of these algorithms in order to utimately realise these gains for our science goals. 


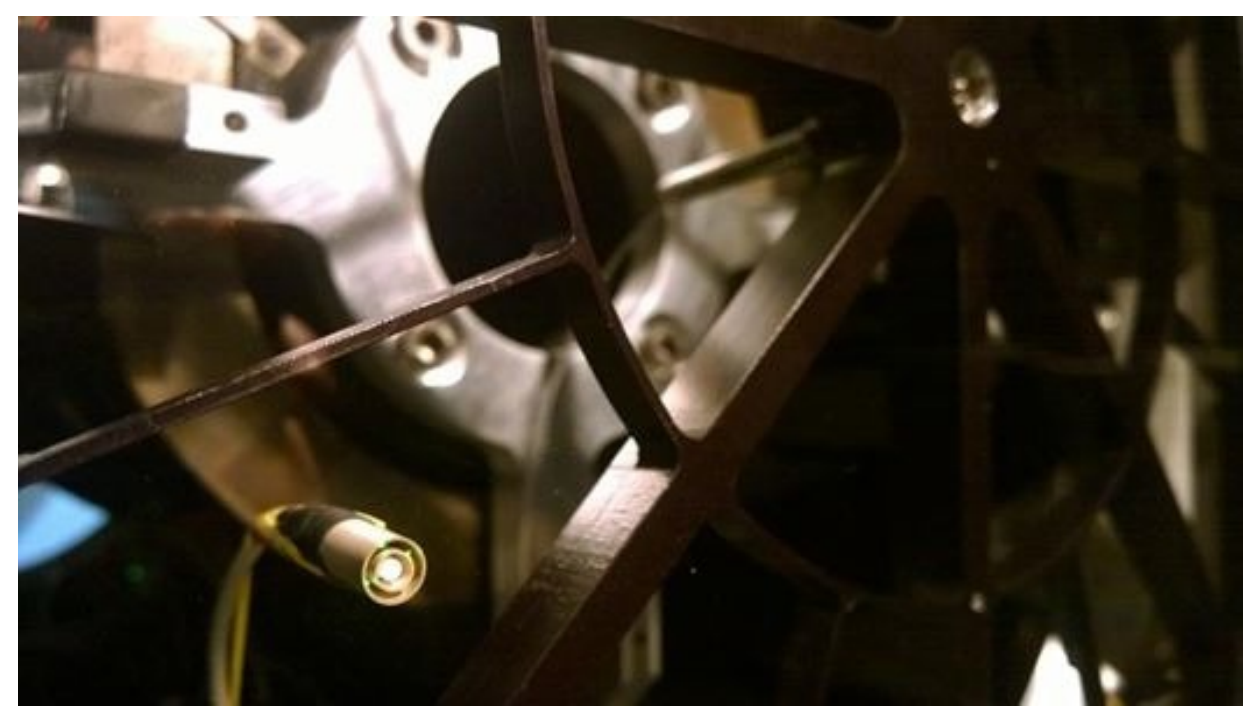

Figure 6. A Proto-TAIPAN Starbug (lower left) installed on the field plate, with elements of the field plate support structure shown as well. Note the concentric piezoceramic tubes, as well as the metrology fibres (three green dots between the two tubes). The electrical power, fibre feed, and vacuum hose extend behind the Starbug before bending downward to connect to the high voltage power source, the imaging CCD, and the vacuum pump, respectively.

One option we are exploring via detailed simulations is to measure the expected reconfiguration time for each Starbug independently, and only move those Starbugs that are in the fastest 90-95\%. We will lose some efficiency due to the reduced number of observed targets, but the tradeoff is potentially a much faster reconfiguration time, a gain that will be of particular benefit to the FunnelWeb survey.

\section{PROTO-TAIPAN ON-SKY TESTS}

In May of 2014, we installed prototype components of the TAIPAN instrument in order to test the installation, operation, and observing procedures. The prototype components included a clear plastic field plate (bent by means of a rigid metal frame to the appropriate radius of curvature of the focal surface), several Starbugs, additional fibres for guiding and fiducial positioning, a metrology camera for positioning and closed-loop motion control of the Starbugs, a vacuum system to ensure Starbug adhesion to the field plate, electronics for both high and low voltage to power cameras, control software, and a variety of other subsidiary systems.

These components passed all of the required tests - the vacuum was sufficient to hold several Starbugs onto the field plate simultaneously, even while the telescope was in motion; the metrology system allowed the Starbug positions to be measured both in static and dynamic configurations, and the Starbugs themselves performed well enough to place large $(1.5 \mathrm{~mm})$ imaging bundle payloads onto pre-selected targets. The Proto-TAIPAN tests culminated in a series of motions of the Starbug using the closed-loop metrology system, during which we located a target in the imaging bundle of the Starbug, moved the Starbug off of the star to its "park" position, and then moved the Starbug back to the pre-determined position of the target. Figure 6 shows one Starbug installed on the field plate, while Figure 7 is a single frame from a video showing the control console with a star centred in the imaging fibre (centre of the console, with an expanded view just left of centre).

In addition to the promising test results of the overall TAIPAN system in general (and the Starbugs in particular), we learned several important lessons from Proto-TAIPAN. Small but significant changes in the Starbug design will be implemented in the final TAIPAN Starbugs, the TAIPAN installation procedure will be modified, and important updates to the metrology hardware and software will be undertaken. After several years of research and development, and a focused period of design refinement, prototyping, and on-sky testing, the TAIPAN team is well prepared for full-scale construction of Starbugs and assembly of the TAIPAN instrument over the next year. This will process will culminate in first light for the TAIPAN instrument in the last quarter of 2015, with the TAIPAN survey commencing in early 2016. 


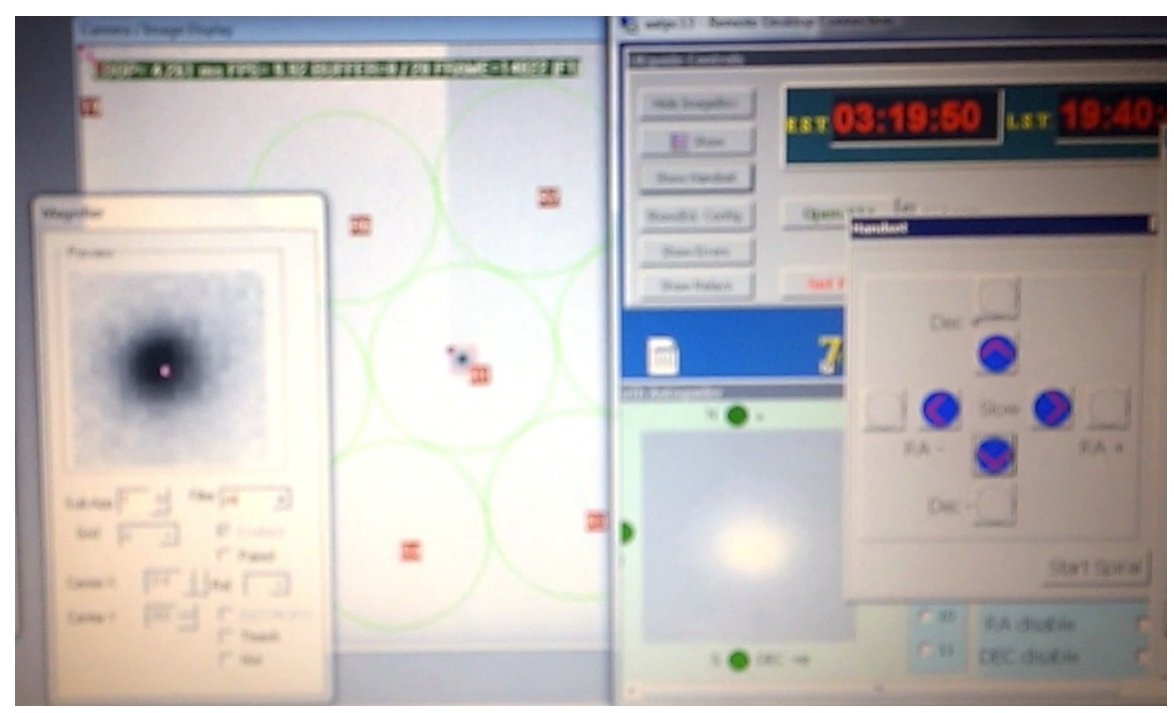

Figure 7. A screen capture of the Proto-TAIPAN control console showing telemetry and guiding (right window), the full view of the imaging fibre bundle carried by the prototype Starbug (centre window), and a zoomed-in view of the star in the centre of the fibre bundle (left window).

\begin{tabular}{|l|c|c|c|c|c|c|c|}
\hline Telescope+Instrument & Diam.(D) & Tel.Field $\left(\phi_{\mathrm{T}}\right)$ & $\operatorname{Inst.Field}\left(\Omega_{\mathrm{I}}\right)$ & $\mathrm{D}^{2}$ & $\mathrm{D}^{4}$ & $\mathrm{~A} \Omega_{\mathrm{T}}$ & $\mathrm{A} \Omega_{\mathrm{I}}$ \\
\hline GMT+MANIFEST & $25.4 \mathrm{~m}$ & $20^{\prime}$ & $314^{\prime}$ & $\equiv 1$ & $\equiv 1$ & $\equiv 1$ & $\equiv 1$ \\
\hline TMT+WFOS & $30 \mathrm{~m}$ & $20^{\prime}$ & $100-200^{\prime}$ & 1.4 & 1.9 & 1.40 & $0.35-070$ \\
\hline E-ELT+DIORAMAS & $42 \mathrm{~m}$ & $10^{\prime}$ & $46^{\prime}$ & 2.7 & 7.5 & 0.68 & 0.40 \\
\hline
\end{tabular}

Figure 8. Comparison of the field size and etendue $(\mathrm{A} \Omega)$ for GMT + MANIFEST and other planned instruments on the proposed Thirty Meter Telescope (TMT) and the European Extremely Large Telescope (E-ELT). Even though the area of the GMT primary mirrors is the smallest of the three, MANIFEST provides the largest etendue of any telescope + instrument combination.

\section{THE FUTURE: MANIFEST}

In addition to facilitating scientific breakthroughs by utilising Starbugs for the TAIPAN survey, the TAIPAN instrument also serves as a testbed for the MANIFEST fibre positioner facility that will be installed on the Giant Magellan Telescope in the middle of the next decade. ${ }^{6,7}$ The entire TAIPAN project (from design, through construction and commissioning, to observation and data analysis) will provide valuable feedback for the hardware and software design of MANIFEST, as well as operational expertise that will be carried over to the GMT. MANIFEST is particularly important to the long-term success of the GMT, for although its collecting area is in fact smaller than the proposed TMT or E-ELT, the GMT + MANIFEST will have the largest etendue of any 30m-class telescope (Figure 8). Furthermore, the Starbugs used in MANIFEST will use a variety of payloads, and they will be able to feed several of the other GMT instruments simultaneously, allowing numerous observational programs to occur in parallel - a potentially revolutionary capability, given that observing time on every $30 \mathrm{~m}$-class telescope is expected to be at a premium. As impressive as the scientific output is expected to be for the other GMT instruments individually, MANIFEST will truly allow it to reach its full potential as a world-leading observational facility. 


\section{REFERENCES}

[1] Croom, S. M., Lawrence, J. S., Bland-Hawthorn, J., Bryant, J. J., Fogarty, L., Richards, S., Goodwin, M., Farrell, T., Miziarski, S., Heald, R., Jones, D. H., Lee, S., Colless, M., Brough, S., Hopkins, A. M., Bauer, A. E., Birchall, M. N., Ellis, S., Horton, A., Leon-Saval, S., Lewis, G., Lpez-Snchez, . R., Min, S., Trinh, C., Trowland, H., "The Sydney-AAO Multi-object Integral field spectrograph", MNRAS 421, 872 (2012).

[2] Eisenstein, D.J., Weinberg, D.H., Agol, E., et al., "SDSS-III: Massive Spectroscopic Surveys of the Distant Universe, the Milky Way, and Extra-Solar Planetary Systems", Astronomical Journal 142 (2011) 72.

[3] Lewis, I. J., Cannon, R. D., Taylor, K., Glazebrook, K., Bailey, J. A., Baldry, I. K., Barton, J. R., Bridges, T. J., Dalton, G. B., Farrell, T. J., Gray, P. M., Lankshear, A., McCowage, C., Parry, I. R., Sharples, R. M., Shortridge, K., Smith, G. A., Stevenson, J., Straede, J. O., Waller, L. G., Whittard, J. D., Wilcox, J. K., and Willis, K. C., "The Anglo-Australian Observatory 2dF facility", MNRAS 333, 279-299 (2002).

[4] Gillingham, P. R., Miziarski, S., Akiyama, M., and Klocke, V., "Echidna: a multifiber positioner for the Subaru prime focus", Proc. SPIE 4008, Optical and IR Telescope Instrumentation and Detectors, 1395-1403 (2000).

[5] Gilbert, J., Goodwin, M., Heijmans, J., Muller, R., Miziarski, S., Brzeski, J., Waller, L. G., Saunders, W., Bennet, A., Tims, J., "Starbugs: all-singing, all-dancing fibre positioning robots", Proc. SPIE 8450, Modern Technologies in Space- and Ground-based Telescopes and Instrumentation II, 84501A (2012).

[6] Goodwin, M., Brzeski, J., Case, S., Colless, M., Farrell, T., Gers, L., Gilbert, J., Heijmans, J., Hopkins, A., Lawrence, J. S., Miziarski, S., Monnet, G., Muler, R., Saunders, W., Smith, G., Tims, J., Waller, L. G., "MANIFEST instrument concept and related technologies", Proc. SPIE 8446, Ground-based and Airborne Instrumentation for Astronomy IV, 84467I (2012).

[7] Lawrence, J., Brown, D., Brzeski, J., Case, S., Colless, M., Farrell, T., Gers, L., Gilbert, J., Goodwin, M., Jacoby, G., Hopkins, A., Kuehn, K., Lorente, N.P.F., Miziarski, S., Muller, R., Nichani, V., Rakman, A., Richards, S., Saunders, W., Staszak, N., Tims, J., Waller, L., "The MANIFEST fibre positioning system for the Giant Magellan Telescope", Proc. SPIE 9147, Ground-based and Airborne Instrumentation for Astronomy $\mathrm{V},(2014)$ 9147-341.

[8] Goodwin, M., Lorente, N. P. F., Hong, S. E., Satorre, C., Lawrence, J., "Field target allocation and routing algorithms for starbugs", Proc. SPIE 9152, Software and Cyberinfrastructure for Astronomy III, in press (2014) 9152-26.

[9] Brown, D. M., Case, S., Gilbert, J., Goodwin, M., Jacobs, D., Kuehn, K., Lawrence, J. S., Lorente, N. P. F., Nichani, V., Saunders, W., Staszak, N., Tims, J., "Starbug fibre positioning robots: performance and reliability enhancements", Proc. SPIE 9151, Advances in Optical and Mechanical Technologies for Telescopes and Instrumentation, in press (2014) 9151-45.

[10] Johnson, S., et al., R. Taylor, R., Bailes, M., Bartel, N., Baugh, C., Bietenholz, M., Blake, C., Braun, R., Brown, J., Chatterjee, S., Darling, J., Deller, A., Dodson, R., Edwards, P., Ekers, R., Ellingsen, S., Feain, I., Gaensler, B., Haverkorn, M., Hobbs, G., Hopkins, A., Jackson, C., James, C., Joncas, G., Kaspi, V., Kilborn, V., Koribalski, B., Kothes, R., Landecker, T., Lenc, A., Lovell, J., Macquart, J.-P., Manchester, R., Matthews, D., McClure-Griffiths, N., Norris, R., Pen, U.-L., Phillips, C., Power, C., Protheroe, R., Sadler, E., Schmidt, B., Stairs, I., Staveley-Smith, L., Stil, J., Tingay, S., Tzioumis, A., Walker, M., Wall, J., Wolleben, M., "Science with ASKAP, the Australian square-kilometre-array pathfinder", Experimental Astronomy, Volume 22, Issue 3, pp.151-273 (2008).

[11] Ricker, G., "Transiting Exoplanet Survey Satellite (TESS)", Proc. SPIE 9143, Space Telescopes and Instrumentation: Optical, Infrared, and Millimeter Wave, in press (2014) 9143-508. 